\title{
The Biomechanical Effects on the Spine during Gait Using Inverse Dynamics
}

\author{
Do-Yun Jeong, Heoung-Jae Chun \\ Department of Mechanical Engineering Yonsei University \\ Seodaemun-guYonesi-ro 50, Seoul, South Korea \\ jjdoyun@gmail.com; hjchun@yonsei.ac.kr
}

\section{Extended Abstract}

Musculoskeletal disorders are health impairments caused by minor damage to muscles and joints because of excessive use of certain body parts or degenerative changes in tissue due to aging. Specifically, low back pain accounts for a large proportion of total musculoskeletal disorders in which spinal stenosis is the major cause of pain [1]. Severe disorders are also caused in the lower extremities due to the push from deformed vertebrae to the peripheral nerves. Although several studies were conducted in the past on the aforementioned-type of low back pain, most of them involved direct experimentation for clinical purposes which costs significant time and space expenses. Therefore, recent studies have focused on constructing a computational model and confirming the expected results via computational analysis. Computational analysis saves time and cost involved in experimentation which can be used to perform an efficient study by minimizing space constraints. In the present study, a computational musculoskeletal model is constructed to proceed with inverse dynamics and analyzed the muscle forces [2] between two groups, namely the group with normal subjects and group with stenosis subjects, using computer.

Ten normal subjects and ten stenosis subjects who were approved by the hospital's institutional review board were recruited and gait experiments were performed. After attaching motion capture markers and EMG sensors to the subjects, the movement for gait analysis was performed to collect kinematic information and muscle activity signals. The kinematic information was input into the computational musculoskeletal model generated by AnyBody S/W [3] and the optimization process for marker positions was performed. Subsequently, muscle activity and muscle force of major muscles were calculated by using inverse dynamic analysis. The calculated muscle activities were compared to the muscle activity signals of the subjects measured through the EMG sensors for verifying the computational model. The calculated and measured signals showed similar tendencies and exhibited almost the same values at the maximum as confirmed in the study. The calculated maximum values for muscle forces in normal and stenosis subjects were analyzed to determine statistically significant differences. The stance and swing phase were separately analyzed. There were significant differences in the erector spinae, psoas major, gluteus medius, and gastrocnemius at the stance phase, and also in the erector spinae and obliquus externus at the swing phase. Therefore, it is concluded that the risk of injury to the muscles of stenosis patient is high, and this should be considered during diagnosis and rehabilitation. By using a computational musculoskeletal model, efficient research can be conducted with minimum time and space constraints.

\section{Acknowledgement}

This research was supported by Basic Science Research Program through the National Research Foundation of Korea (NRF) funded by the Ministry of Science and ICT (NRF-2016R1A2B3012850).

\section{References}

[1] S. Hall, J. D. Bartleson, B. M. Onofrio, H. L. Baker, H. Okazaki, D. O’Duffy, "Lumbar Spinal Stenosis: Clinical features, Diagnostic procedures, and results of surgical treatment in 68 patients," Annals of Internal Medicine, vol. 103, pp. 271-275, 1985.

[2] M. Damsgaard, J. Rasmussn, S. T. Christensen, E. Surma, M. Zee, "Analysis of Musculoskeletal Systems in the AnyBodyModelingSystem," Simulation Modelling Practice and Theory., vol. 14, no. 8, pp. 1100-1111, 2006.

[3] M. Zee, L. Hansen, C. Wong, J. Rasmussen, E.B. Simonsen, "A Generic Detailed Rigid-Body Lumbar Spine Model," Journal of Biomechanics.,vol. 40, no. 6, pp. 1219-1227, 2007. 